\title{
High performance microfluidic rectifiers for viscoelastic fluid flow
}

\author{
Patrícia C. Sousa, ${ }^{a}$ Fernando T. Pinho, ${ }^{b}$ Mónica S. N. Oliveira ${ }^{a}$ and Manuel A. Alves*a
}

Received 27th September 2011, Accepted 30th September 2011

DOI: $10.1039 / \mathrm{c} 1 \mathrm{ra00803j}$

The flow of Newtonian and non-Newtonian fluids within microfluidic rectifiers with a hyperbolic shape was investigated to assess the effect of the bounding walls on the diodicity of the microfluidic device and achieve high flow anisotropy. Three microchannels were used, with different depths and the same geometrical configuration, which creates a strong extensional flow and generates high anisotropic flow resistance between the two flow directions. The Newtonian fluid, de-ionized water, was used as a reference fluid. The viscoelastic fluid used was an aqueous solution of polyethylene oxide $(0.1 \% \mathrm{w} / \mathrm{w})$ with high molecular weight. The flow patterns were visualized using streak photography and the velocity field was investigated using micro-particle image velocimetry.

Moreover, pressure drop measurements were performed in order to compare the diodicity achieved in the microfluidic rectifiers. For the Newtonian fluid flow, the experimental results are compared with numerical predictions obtained using a finite-volume method and good agreement was found between both. For the viscoelastic fluid, significant anisotropic flow resistance can be achieved. The effect of the bounding walls is analysed and found to be qualitatively similar for all microchannels.

Nevertheless, in quantitative terms, the diodicity is enhanced when the wall effect is reduced, i.e. when the channels are deeper. A maximum diodicity above six was found for the deeper channel, a value

30 well beyond those previously reported.

\section{Introduction}

Microfluidic devices are systems which operate with small amounts of fluids and have integrated channels, whose dimensions are of the order of tens to hundreds of microns. One current challenge of engineering science is to scale-down (process intensification) in order to achieve integrated and portable "lab-on-a-chip" tools able to operate with high resolution and

40 sensitivity, short analysis time, low cost and reduced volumes of reagents and waste. ${ }^{1}$ Microfluidic technology is applied across fields: in biology for cellular analysis or DNA separation; in medicine for clinical diagnostics; in chemistry to undertake chemical reactions. $^{2-5}$

The small length scales that characterize these microsystems lead typically to low Reynolds numbers $(R e)$ and consequently laminar flow conditions. Furthermore, surfaces forces, which are often negligible in flows at the macroscale, may become significant in microfluidics due to the strong intermolecular forces. ${ }^{6}$ The fluids of interest in such lab-on-a-chip applications have often non-Newtonian rheological behaviour, as for example

${ }^{a}$ Departamento de Engenharia Química, CEFT, Faculdade de Engenharia da Universidade do Porto, Rua Dr Roberto Frias, 4200-465, Porto, Portugal.E-mail:mmalves@fe.up.pt Fax+351225081449;

Tel: +351225081680

${ }^{b}$ Departamento de Engenharia Mecânica, CEFT, Faculdade de Engenharia da Universidade do Porto, Rua Dr Roberto Frias, 4200-465, Porto, Portugal. saliva, ${ }^{7}$ blood, ${ }^{8}$ several suspensions or other fluids containing small amounts of polymers. 9,10

The non-linear rheological properties of these fluids result in complex flow phenomena, which need to be better understood in order to optimize the design of microfluidic devices.

Viscoelastic fluid flow is prone to instabilities, which in microfluidics are often purely elastic in nature as inertial effects are small. These purely elastic instabilities, as documented in Muller et al. ${ }^{11}$ and Larson et al. ${ }^{12}$ for Taylor-Couette flow, occur above a critical Deborah number $(D e)$ and result from the combination of curved streamlines and development of large normal stresses. Subsequently, Pakdel and McKinley ${ }^{13}$ proposed a criterion to predict the critical conditions for the onset of purely elastic instabilities.

Utilizing a microfluidic cross-slot geometry, Arratia et al. ${ }^{14}$ observed experimentally that a viscoelastic fluid under creeping flow conditions can undergo different types of instabilities. These authors reported the onset of a first instability, in which the flow becomes asymmetric but remains steady, followed by a second instability, in which the flow becomes unsteady, with the amplitude of oscillation increasing with the flow rate. Subsequently, Poole et $a l .{ }^{15}$ demonstrated that the steady asymmetry observed in the cross-slot geometry can be predicted numerically for creeping flow conditions, using the simplest differential viscoelastic model, the upper-convected Maxwell (UCM) model. Broad attention has been paid recently to geometries in which viscoelastic effects emerge in highly elongational fields, such as the cross-slot, ${ }^{16}$ T-shaped geometries ${ }^{17}$ and flow-focusing devices. ${ }^{18}$ 
1 Elastic instabilities can be useful to promote mixing at low Reynolds number flows. ${ }^{19}$ In fact, purely elastic instabilities stimulate a disordered state of the flow brought about by elastic stresses even at vanishingly small $R e$, giving rise to the concept of elastic turbulence. ${ }^{20,21}$ With the fluid motion occurring over a wide range of temporal and spatial scales, elastic turbulence generates high flow resistance and increases the rate of mixing. ${ }^{22}$

Taking advantage of elastic nonlinearities, Groisman and coworkers $^{23,24}$ developed several ingenious microfluidic devices which operate as control and memory elements. These authors exploited the viscoelastic rheological properties of dilute polymeric fluids and found that increasing the applied pressure gradient lead to complex flow behaviour, due to the differences between coil stretching and relaxation, and consequently the variation of the flow rate was found to be small for a wide range of applied pressures. ${ }^{25} \mathrm{~A}$ microfluidic rectifier with no moving parts was also developed, making use of the nonlinearities brought about by elastic stresses in viscoelastic fluid flow. ${ }^{24}$ In the proposed microfluidic device, consisting of a series of triangular cavities, the non-Newtonian fluid experiences a different deformation history when flowing in the two possible flow directions, leading to a significant anisotropic flow resistance. The development of efficient flow rectifiers is an important area of research in microfluidics, namely to be used in the development of piezoelectric-driven micropumps. The technology of microscale pumping has been the aim of several investigations with applications as diverse as drug delivery systems, fuel delivery in micro-engines and cooling in microelectronics. $^{25}$ However, despite the interest of non-Newtonian fluid flows in microfluidics, in these studies, the flow of Newtonian fluids has been much more frequently investigated and studies of microfluidic rectifiers using viscoelastic fluids are still scarce. ${ }^{24,26,27}$

With the aim of designing more efficient microfluidic rectifiers for viscoelastic fluids that are able to operate even under creeping flow conditions, we presented previously a comparison between the efficiency of distinct geometries, namely the triangular nozzle/diffuser rectifier proposed in the literature and a new rectifier based on hyperbolic-shaped elements. ${ }^{27} \mathrm{~A}$ detailed investigation, in terms of pressure drop measurements and visualizations of the flow patterns for different viscoelastic fluids as well as for a Newtonian reference fluid, was carried out. In that work, hyperbolic-shaped geometries were chosen based on the work of Oliveira et al. ${ }^{28}$ in order to generate a quasi-ideal extensional flow and the device with a Hencky strain $\varepsilon_{H}=2$ was identified to exhibit the highest diodicity. Additionally, the importance of wall effects imposed by the shallow depth of the microchannels and its influence on the diodicity arose as an important topic for further research. Therefore, here, we investigate the effect of channel depth on the diodicity using the most efficient hyperbolic shaped microfluidic rectifier presented earlier. ${ }^{27}$ For this purpose, we use three microchannels with different depths, $h=46 \mu \mathrm{m}, 88 \mu \mathrm{m}$ and $120 \mu \mathrm{m}$. Pressure drop measurements in addition to flow visualization and microparticle image velocimetry ( $\mu$ PIV) measurements were carried out for a wide range of flow rates. The non-Newtonian fluid used here is an aqueous solution of a high molecular weight polyethylene oxide (PEO), with molecular weight $M_{w}=8 \times$ $10^{6} \mathrm{~g} \mathrm{~mol}^{-1}$, which presented the highest rectification effect of all the fluids tested previously. ${ }^{27}$ Moreover, the flow of a Newtonian fluid (de-ionized water) is also investigated in order to validate the experimental techniques and to be used as reference.

\section{Experimental}

\subsection{Experimental techniques}

The three microchannels used in the experiments were designed to have the same generic geometrical shape but different depths (h).

The devices, fabricated in polydimethylsiloxane (PDMS) using standard soft-lithography, ${ }^{29}$ include two inlets/outlets located at the extremes of the channels and two pressure taps on each side of the test section of the microgeometry. In Fig. 1(a) we show an optical transmission microscope image of a typical microchannel

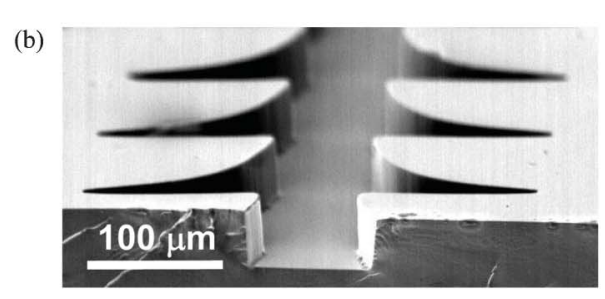

(c)

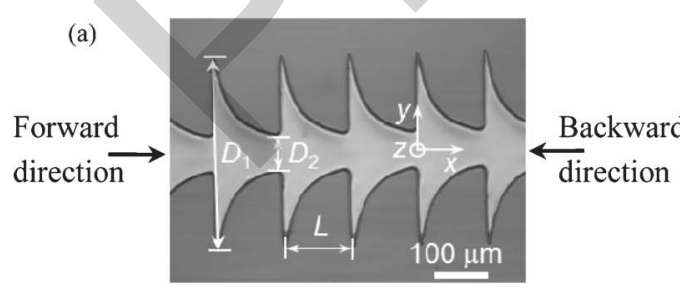

(d)

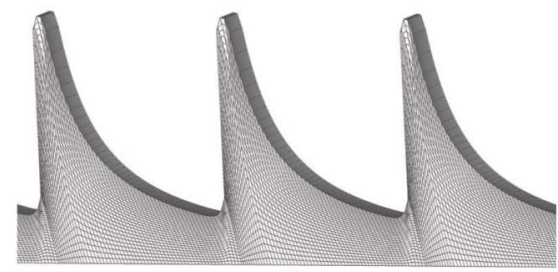

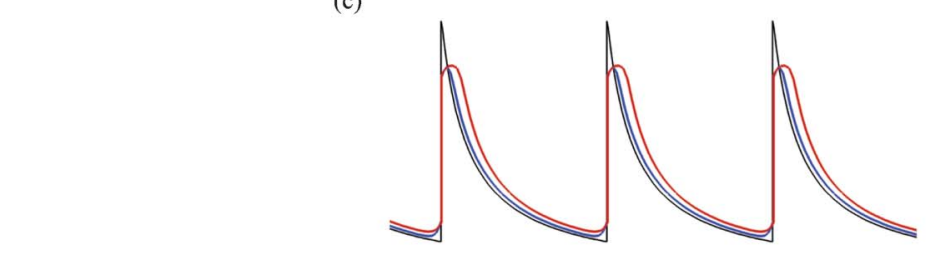

Fig. 1 (a) Optical micrograph of the hyperbolic microfluidic rectifier A $(h=46 \mu \mathrm{m})$. Microchannels B and C have the same geometrical shape but have depths of $88 \mu \mathrm{m}$ and $120 \mu \mathrm{m}$, respectively. (b) SEM image illustrating the verticality of the walls of microchannel A. Only part of the microchannels is shown. (c) Comparison between the designed shape of the microchannel (thin black line), the real shape of the microchannel A ( $h=46 \mu \mathrm{m}$, blue line) and the real shape of microchannels B and C with $88 \mu \mathrm{m}$ and $120 \mu \mathrm{m}$ in depth, respectively (red line). Note that the shape of microchannels B and C are similar. (d) Zoomed view of the coarse mesh used in the numerical simulation of the Newtonian fluid flow in microchannel A. 
used in the experiments. A scanning electron microscopy (SEM) image of the $46 \mu \mathrm{m}$ deep microchannel is also shown in Fig. 1(b) to illustrate the well-defined and quasi-vertical walls of the PDMS microchannels. The general hyperbolic function that was used to describe the shape of the channels is $y= \pm a /(1+b x)$, with $a=200 \mu \mathrm{m}$ and $b=0.05 \mu^{-1} ; x$ is the axial position $(0 \leqslant x / \mu \mathrm{m} \leqslant L)$, where $L$ is the length of each hyperbolic element $(L=128 \mu \mathrm{m})$. Each microfluidic diode is composed of 42 repetitive hyperbolic elements. The chrome mask used in the 0 manufacturing process follows closely this hyperbolic shape and was used to make the moulds from which the channels were produced, which in principle would only differ in depth. However, the actual moulds are slightly different due to inaccuracies inherent to the photolithography fabrication procedure and this results in small differences in the shape of the final PDMS channels as shown in Fig. 1(c). The corresponding characteristic dimensions of the channels are given in Table 1, including the total Hencky strain $\left(\varepsilon_{H}\right)$, defined as $\varepsilon_{H}=\ln \left(D_{1} / D_{2}\right)$.

The shape of the microchannels follows closely the following curves:

$$
\begin{aligned}
& \text { Channel A }(h=46 \mu \mathrm{m}) \\
& \begin{cases}|y / \mu \mathrm{m}|=153.6+2.3 \sqrt{4^{2}-(x-4)^{2}} & 0<x / \mu \mathrm{m} \leq 7.7 \\
|y / \mu \mathrm{m}|=\frac{220}{1+0.052 x}+15 \mathrm{e}^{(x-128) / 3} & 7.7<x / \mu \mathrm{m} \leq 128\end{cases}
\end{aligned}
$$

Channel B $(h=88 \mu \mathrm{m})$ and channel C $(h=120 \mu \mathrm{m})$

$$
\left\{\begin{array}{c}
|y / \mu \mathrm{m}|=155.5+\sqrt{7.5^{2}-(x-7.5)^{2}} \quad 0<x / \mu \mathrm{m} \leq 14.9 \\
|y / \mu \mathrm{m}|=\frac{380}{1+0.097 x-9.1 \times 10^{-5} x^{2}}+10 \mathrm{e}^{(x-128) / 4} \\
14.9<x / \mu \mathrm{m} \leq 128
\end{array}\right.
$$

The configuration of the experimental set-up used in this work 40 is identical to that employed in a previous work. ${ }^{27}$ Flow is imposed by means of a syringe pump (PHD2000, Harvard Apparatus), used to feed the fluid to the channels and control the volumetric flow rate.

For pressure drop measurements, the pressure taps in 45 the microchannel were connected to a pressure transducer (Honeywell, model 26PC series) in order to measure the differential pressure drop inside the test section. A $12 \mathrm{~V} \mathrm{DC}$ power supply (Lascar electronics, PSU 206) was used to power the pressure sensors that were also connected to a computer, via

Table 1 Dimensions of the hyperbolic microgeometries and resulting

\begin{tabular}{|c|c|c|c|c|c|c|c|}
\hline \multicolumn{3}{|c|}{ Projected (chrome mask) } & \multirow[b]{2}{*}{$\begin{array}{l}L \\
{[\mu \mathrm{m}]}\end{array}$} & \multicolumn{4}{|c|}{ PDMS microchannel } \\
\hline $\begin{array}{l}D_{1} \\
{[\mu \mathrm{m}]}\end{array}$ & $\begin{array}{l}D_{2} \\
{[\mu \mathrm{m}]}\end{array}$ & $\varepsilon_{H}$ & & $\begin{array}{l}h \\
{[\mu \mathrm{m}]}\end{array}$ & $\begin{array}{l}D_{1} \\
{[\mu \mathrm{m}]}\end{array}$ & $\begin{array}{l}D_{2} \\
{[\mu \mathrm{m}]}\end{array}$ & $\varepsilon_{H}$ \\
\hline 400 & 54 & 2.0 & 128 & 46 & 326 & 63 & 1.64 \\
\hline 400 & 54 & 2.0 & 128 & 88 & 326 & 70 & 1.54 \\
\hline 400 & 54 & 2.0 & 128 & 120 & 326 & 70 & 1.54 \\
\hline
\end{tabular}
Hencky strain values a data acquisition card (NI USB-6218, National Instruments). The output data was recorded using LabView v7.1. The pressure sensors were calibrated using a static column of water or using a compressed air line depending on the pressure range. A detailed description can be found in Sousa et al. ${ }^{27}$

Visualizations of the flow patterns and measurements of the velocity field were performed at the centreplane of the microchannel i.e. at mid-distance between top and bottom planar bounding walls. The imaging experimental set-up is composed of an inverted epi-fluorescence microscope (DMI 5000M, Leica Microsystems $\mathrm{GmbH}$ ) equipped with a filter cube (Leica Microsystems $\mathrm{GmbH}$, excitation filter BP 530-545 nm, dichroic $565 \mathrm{~nm}$ and barrier filter $610-675 \mathrm{~nm}$ ) as well as with a light source, objective and digital camera appropriate for each optical technique used.

For visualizations of the flow patterns, we employed streak photography. For this, the light source used was a $100 \mathrm{~W}$ mercury lamp. The fluids were seeded with $1 \mu \mathrm{m}$ fluorescent tracer particles (Nile Red, Molecular Probes, Invitrogen, Ex/Em: $520 / 580 \mathrm{~nm}$ ) and the streak images were captured through a $10 \times(\mathrm{NA}=0.25)$ objective lens using a CCD camera (DFC350 FX, Leica Microsystems GmbH).

For micro-particle image velocimetry measurements, the fluorescent particles used are $0.5 \mu \mathrm{m}$ in diameter and the light source was a double-pulsed $532 \mathrm{~nm} \mathrm{Nd:YAG} \mathrm{laser} \mathrm{(Dual} \mathrm{Power}$ 65-15, Dantec Dynamics). Images were captured using a $20 \times$ $(\mathrm{NA}=0.4)$ microscope objective and a CCD digital camera (Flow Sense 4M, Dantec Dynamics), with a resolution of $2048 \times$ 2048 pixels and running on double frame mode. The time interval between pulses was adjusted so that the displacement of the particles between the two consecutive frames was about $25 \%$ of the width of the interrogation area. Interrogation areas of $64 \times 32$ pixels, with a $50 \%$ overlap were used to create the vector maps. For steady flow, the vector maps were obtained by ensemble averaging 150 pairs of images. On the other hand, for unsteady flow, we present both instantaneous and ensembleaverage vector maps obtained from a set of 1000 pairs of images.

The depth over which there is a contribution of unfocused particles to the velocity field determined by $\mu \mathrm{PIV}$ is called the total measurement depth, $\delta z_{m}$, and can be calculated as the sum of three different effects: the effect due to diffraction, the effect due to geometrical optics and the effect due to the finite size of the particle, ${ }^{30}$ respectively corresponding to the first, second and third terms on the right-hand-side of Eqn (3):

$$
\delta z_{m}=\frac{3 n \lambda_{0}}{(\mathrm{NA})^{2}}+\frac{2.16 d_{p}}{\tan \theta}+d_{p}
$$

where $n$ is the refractive index, $\lambda_{0}$ is the wavelength of the light (in vacuum), NA is the numerical aperture of the microscope objective and $d_{p}$ is the seeding particle diameter. For the streak imaging optical set-up, the measurement depth is $\delta z_{m}=37 \mu \mathrm{m}$, whereas for the $\mu$ PIV set-up, $\delta z_{m}=14 \mu \mathrm{m}$.

\subsection{Fluid rheology}

The fluids used in this investigation consist of a Newtonian fluid (de-ionized water) and a non-Newtonian fluid, which is an aqueous solution of a high molecular weight polyethylene oxide 
$1 \quad\left(\mathrm{PEO}, M_{\mathrm{w}}=8 \times 10^{6} \mathrm{~g} \mathrm{~mol}^{-1}\right.$, Sigma-Aldrich) at $0.1 \%(\mathrm{w} / \mathrm{w})$ concentration. The density of the viscoelastic fluid, measured at the reference temperature $\left(T_{0}=293.2 \mathrm{~K}\right)$, is $\rho=998 \mathrm{~kg} \mathrm{~m}^{-3}$.

The rheological properties of the viscoelastic solution were characterized in steady shear and in uniaxial extension flow and are presented in Sousa et al., ${ }^{27}$ where a thorough description of the experimental rheological characterization and techniques can be found. Here, only the most relevant results are presented. The characteristic relaxation time of the viscoelastic fluid $(\lambda)$, 0 measured at the reference temperature using a capillary break-up extensional rheometer (CaBER), was found to be $73.9 \mathrm{~ms}$.

Shear rheology measurements were performed on a rotational rheometer (Physica MCR301, Anton Paar) using a cone-plate geometry with $75 \mathrm{~mm}$ diameter and $1^{\circ}$ angle in the temperature range of $283.2 \leqslant T / \mathrm{K} \leqslant 303.2$. The flow curves obtained experimentally were shifted to the reference temperature $\left(T_{0}=\right.$ 293.2 K) using the time-temperature superposition method in order to obtain the master curve and the temperature influence on the shift factor, $a_{T}$, generally defined as: ${ }^{31}$

$$
a_{T}=\frac{\eta(T)}{\eta\left(T_{0}\right)} \frac{T_{0}}{T} \frac{\rho_{0}}{\rho}
$$

where $\eta\left(T_{0}\right)$ is the shear viscosity at the reference temperature $T_{0}, \eta(T)$ is the shear viscosity at a given temperature $T$ and $\rho_{0}$ and $\rho$ are the fluid densities at temperatures $T_{0}$ and $T$, respectively.

Fig. 2 shows the master curve obtained for the viscoelastic fluid, which was fitted using the simplified form of the singlemode linear Phan-Thien-Tanner (sPTT) model $^{32}$ with a solvent contribution. The parameters obtained were: extensibility parameter, $\varepsilon=0.04$; polymer viscosity coefficient, $\eta_{P}=0.0045 \mathrm{~Pa} \mathrm{~s}$; relaxation time, $\lambda=73.9 \mathrm{~ms}$ (determined from CaBER measurements); Newtonian solvent viscosity, $\eta_{S}=0.003 \mathrm{~Pa}$ s. More details can be found in Sousa et al. ${ }^{27}$ The flow curve was also fitted using the Carreau-Yasuda model, ${ }^{33}$ which is able to

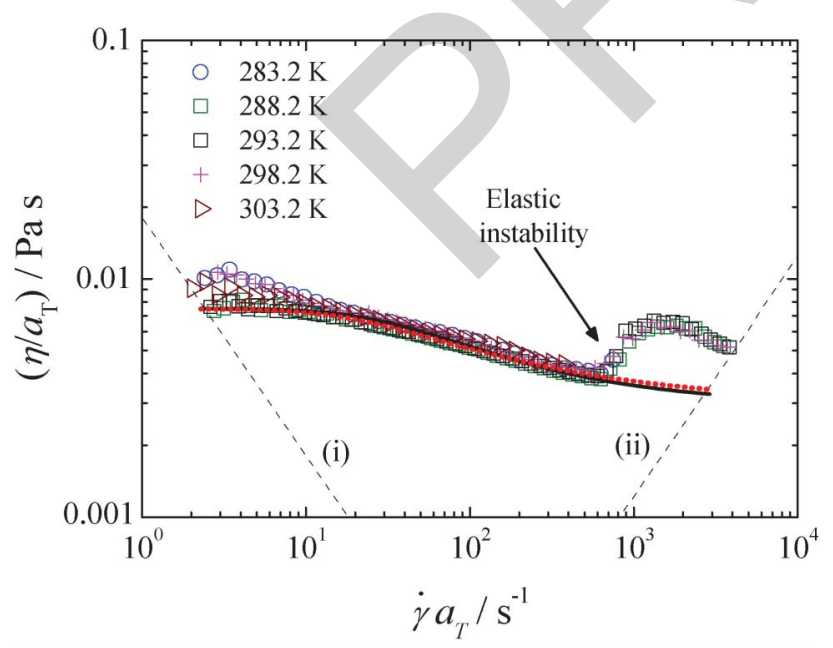

Fig. 2 Master curve of the steady shear viscosity for the viscoelastic fluid (symbols) at the reference temperature $\left(T_{0}=293.2 \mathrm{~K}\right)$. The solid and the dotted lines represent the fit of the sPTT and Carreau-Yasuda models to the experimental data, respectively. The dashed straight lines represent the minimum measurable shear viscosity determined from $20 \times$ the minimum measurable torque of the rheometer (i) and the onset of secondary flow due to Taylor instabilities (ii). model the fluid shear-thinning behaviour, but not its elasticity: $\eta=\eta_{\mathrm{S}}+\left(\eta_{0}-\eta_{\mathrm{S}}\right) /\left[1+(\Lambda \dot{\gamma})^{\mathrm{a}}\right]^{(1-n) / a}$. The corresponding parameters are: $\eta_{0}=0.0075 \mathrm{~Pa} \mathrm{~s}, \eta_{\mathrm{S}}=0.003 \mathrm{~Pa} \mathrm{~s}, \Lambda=0.05 \mathrm{~s}, n=0.53$ and $a=1.8$. The predictions of both models are shown in Fig. 2, and this information can be useful, for example, for future numerical calculations.

\section{Numerical}

\subsection{Governing equations and numerical method}

The Newtonian fluid flow was simulated numerically using a fully-implicit finite-volume method with a time marching pressure-correction algorithm. ${ }^{34}$ The governing equations that describe an isothermal, laminar and incompressible fluid flow are those of conservation of mass and momentum:

$$
\begin{aligned}
\nabla \cdot \mathbf{u} & =0 \\
\rho\left[\frac{\partial \mathbf{u}}{\partial t}+\mathbf{u} \cdot \nabla \mathbf{u}\right] & =-\nabla p+\nabla \cdot \tau
\end{aligned}
$$

where $p$ is the pressure and the extra-stress tensor $(\tau)$ is defined as the sum of the contribution of a solvent and a polymeric solute $\left(\tau=\tau_{\mathbf{s}}+\tau_{\mathbf{p}}\right.$ ). Since in this work we will restrict the numerical simulations to Newtonian fluid flows, the polymeric contribution to the extra-stress tensor is null $\left(\tau_{\mathbf{p}}=0\right)$ and the stress-tensor is calculated considering only the solvent contribution:

$$
\tau_{s}=\eta(\dot{\gamma})\left(\nabla \mathbf{u}+\nabla \mathbf{u}^{\mathrm{T}}\right)
$$

where $\eta(\dot{\gamma})$ can be either constant (Newtonian fluid, $\eta(\dot{\gamma})=\eta_{\mathrm{S}}$ ) or shear-rate dependent for a generalized Newtonian fluid (GNF), such as the Carreau-Yasuda, in which $\eta(\dot{\gamma})$ is a function of the shear rate, defined as $\dot{\gamma}=\sqrt{I I(\dot{\gamma}) / 2}$, where $I I(\dot{\gamma})$ represents the second invariant of the shear rate tensor, $\dot{\gamma}=\nabla \mathbf{u}+\nabla \mathbf{u}^{\mathrm{T}}$.

In the numerical method, the governing eqn (5) and (6) are integrated in time over a small time step $(\delta t)$ and in space over the control volumes of the computational mesh. Regarding the accuracy of the calculations, the time derivative is discretized using an implicit first-order Euler scheme, the diffusive terms are discretized using second-order central differences and a highresolution scheme, CUBISTA ${ }^{35}$ is used for the discretization of the advective terms. More details of the numerical method used can be found in Oliveira et $a l .{ }^{34}$ and Alves et al. ${ }^{35}$

\subsection{Computational meshes and dimensionless numbers}

We performed numerical simulations of the Newtonian fluid flowing in the forward and backward flow directions of the hyperbolic shaped rectifier. The meshes used to describe the computational domain are three-dimensional, block-structured and are composed of non-uniform cells. Due to symmetry of the Newtonian fluid flow under steady flow conditions, observed in the experiments, symmetry boundary conditions were imposed at the two centreplanes ( $x=0$ and $z=0$ ), thus only a quarter of the total domain was used in the numerical simulations. The computational domain consists of two long straight channels that define the inlet and outlet channels and 10 hyperbolic elements which define the test section and have dimensions matching exactly those used in the experiments. As explained previously in Section 2.1, the fabricated microchannels present 
1 slight differences relative to the idealized hyperbolic shape, due to inaccuracies inherent to the fabrication procedure. As such, the numerical simulations were carried out in the computational domains corresponding to the real geometries described by eqn (1) and(2), rather than the idealized shape printed on the chrome mask [Fig. 1(c)]. We also note that the experimental geometries consist of 42 repeating hyperbolic units, while in the numerical simulations only 10 elements were used in order to reduce the size of the meshes and reduce the computational times. The 0 numerical results confirm that for all flow conditions investigated numerically, the flow field in the hyperbolic elements located in the middle of the computational domain is already fully-developed, and therefore using only 10 elements in the numerical simulations is enough to obtain accurate results since the comparisons are made on the basis of dimensionless quantities. A zoomed perspective of the mesh used for the simulations with channel A is illustrated in Fig. 1(d). Moreover, the Newtonian fluid flow in channel A was simulated using another mesh, with higher refinement level. For channel A, mesh 20 M1 has a total of 290304 cells, while mesh M2 has a total number of 2322432 cells, and was created by doubling the number of cells in each direction. For channels B and C, only mesh M1 was used, also consisting of 290304 cells.

Except for the (inlet/outlet) boundary conditions, the meshes used for the forward and backward flow directions are similar in their characteristics. The inlets and outlets were positioned far from the hyperbolic elements that define the test section allowing fully developed flow conditions to be attained well upstream and downstream of the hyperbolic elements. No-slip boundary 0 conditions were imposed at the solid walls, and at the inlet, a uniform velocity profile was imposed. At the outflow boundary, vanishing streamwise gradients of velocity components are imposed and pressure is linearly extrapolated from the two upstream cells.

Regarding the relevant dimensionless numbers, the aspect ratio of the channel is defined as $A R=h / D_{2}$. The Newtonian fluid flow is also characterized using the Reynolds number, $R e=$ $\rho U_{2} D_{2} / \eta_{0}$, based on the flow characteristics at the contraction throat, where $U_{2}$ is the average velocity, $D_{2}$ is the width [cf. 40 Fig. 1(a)] and $\eta_{0}$ is the fluid shear viscosity. For the viscoelastic fluid flow, the zero-shear rate viscosity $\left(\eta_{0}=\eta_{P}+\eta_{s}\right)$ is used in the definition of the Reynolds number. Additionally, to characterize the viscoelastic fluid flow we use the Deborah number $(D e)$, also based on the flow characteristics at the throat: $45 D e=\lambda U_{2} /\left(D_{2} / 2\right)$. The relaxation time and the shear viscosity used in computation of $D e$ and $R e$ are evaluated at the temperature of the experiments.

The diodicity, which is used to characterize the rectification effects, is defined as the ratio between the pressure drop in the 0 forward direction, $\Delta P_{\text {Forward }}$, and the pressure drop in the

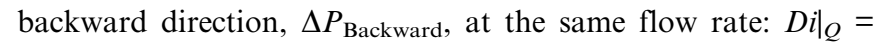
$\Delta P_{\text {Forward }} / \Delta P_{\text {Backward }}$.

\section{Results and discussion}

\subsection{Flow patterns}

The Newtonian fluid flow patterns observed in hyperbolic shaped rectifiers, including rectifier $\mathrm{A}$, were discussed in detail in Sousa et al. ${ }^{27}$ and therefore are not repeated here. For
Newtonian fluid flow through rectifier $\mathrm{B}$ and $\mathrm{C}$, the flow patterns observed are qualitatively similar to those in rectifier A and in these three channels the diodicity is nearly unitary for the range of flow rates investigated (i.e. no rectification effects are observed). The flow phenomena observed in the viscoelastic fluid flow through the hyperbolic shaped microfluidic rectifiers A, B and $\mathrm{C}$ is qualitatively similar. Streak images obtained in rectifier $\mathrm{B}(A R=1.26, h=88 \mu \mathrm{m})$ are shown in Fig. 3. At low flow rates, Newtonian-like flow patterns are observed, exhibiting recirculations inside the hyperbolic corner for both flow directions [Fig. 3(a); forward flow in the first row and backward flow in the second row]. Increasing the flow rate, a distinct flow behaviour is found for the two flow directions, as documented previously in Sousa et $a{ }^{27}$ For the forward direction, the flow becomes asymmetric and unsteady due to onset of elastic instabilities, and recirculations appear and disappear randomly within the hyperbolic elements [cf. Fig. 3(b) in the first row]. In the previous work with the $46 \mu \mathrm{m}$ depth microfluidic rectifier, the onset of these purely elastic instabilities was linked to the appearance of rectification effects. ${ }^{27}$ In that work, a nominal depth of $50 \mu \mathrm{m}$ was assumed, but subsequent SEM measurements of the microchannels showed that the correct depth is $46 \mu \mathrm{m}$, as used in this work. Also, in Sousa et al., ${ }^{27}$ the throat width of the chrome mask was assumed in the calculations while in this work we use the real minimum width of the final PDMS channel, as reported in Table 1. These corrections in the microchannel depth and width lead to an increase of less than $10 \%$ in $R e$ and a decrease of less than $20 \%$ in $D e$ values relative to those reported in the previous work. The rectification effects emerge ( $c f$. Section 4.3) following the appearance of elastic instabilities [Fig. 3(b) in forward flow]. For the backward flow direction, recirculations are visible near the hyperbolic corners and the flow remains steady as the flow rate (or $D e$ ) is increased until a critical $D e$ is reached. The critical value for the appearance of elastic instabilities that incite unsteady flow in the backward direction is significantly higher than in the forward flow direction.

In summary, similar flow behaviour is found for the three microfluidic diodes (A, B and $\mathrm{C}$ ), but the onset of elastic instabilities with the critical Deborah number $\left(D e_{\mathrm{c}}\right)$ increasing with the aspect ratio: while for the microchannel A $(A R=0.73$, $h=46 \mu \mathrm{m})$ elastic instabilities are already evident at $D e_{\mathrm{c}} \approx 20$ in the forward flow direction, for the microchannel $\mathrm{B}(A R=$ 1.26, $h=88 \mu \mathrm{m})$ and microchannel $\mathrm{C}(A R=1.71, h=120 \mu \mathrm{m})$, these are delayed to $D e_{\mathrm{c}} \approx 25$ and $D e_{\mathrm{c}} \approx 45$, respectively.

\section{Velocity field}

\subsection{Newtonian fluid flow-experimental validation}

The velocity field of the Newtonian fluid flow in all hyperbolic rectifiers was determined using $\mu \mathrm{PIV}$ for different flow rates. Fig. 4 shows profiles of the dimensionless axial velocity along the centreline of the microfluidic rectifiers with a depth of $46 \mu \mathrm{m}$ (rectifier A), for both flow directions. Furthermore, the experimental results are compared with the numerical predictions using meshes M1 and M2 and the velocity profiles are split in two regions: one region for a low $R e$ and another region for a higher $R e$. As expected, the velocity profiles along the centreline show a periodic behaviour caused by the geometrical shape of 

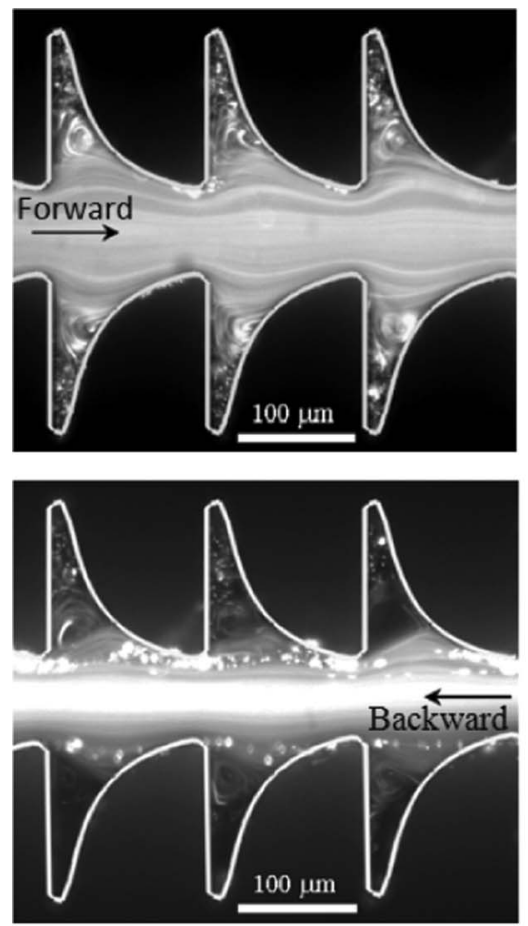

(a) $R e=0.070, D e=12.9$
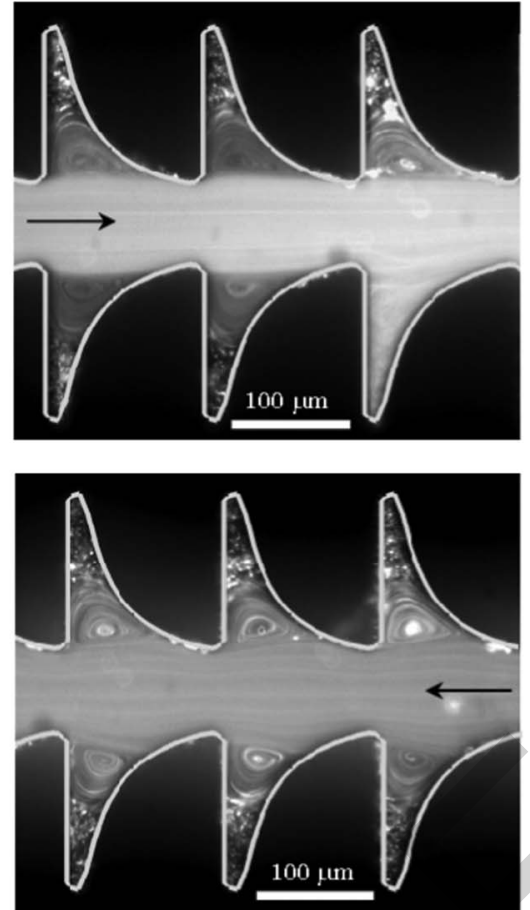

(b) $R e=0.23, D e=43.1$
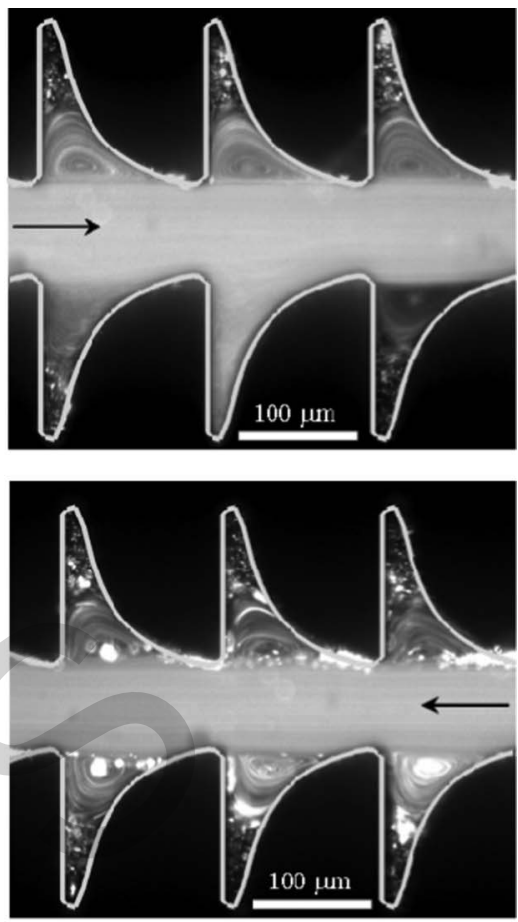

(c) $R e=0.70, D e=129$

10

15

20

Fig. 3 Streak images of the viscoelastic fluid flow in rectifier $\mathrm{B}(A R=1.26 ; h=88 \mu \mathrm{m})$ at different flow rates.

the microchannel, i.e. the sequence of identical hyperbolic elements composed of contractions. As such, the axial velocity varies from a minimum value at a position near the centre of each element, to a maximum value near the contraction region. As such, the axial velocity varies from a minimum value at a position near the centre of each element, to a maximum value near the contraction region. At low flow rates (low $R e$ ), inertia is negligible and the axial velocity profiles for both flow directions collapse due to the reversibility of Newtonian creeping flows

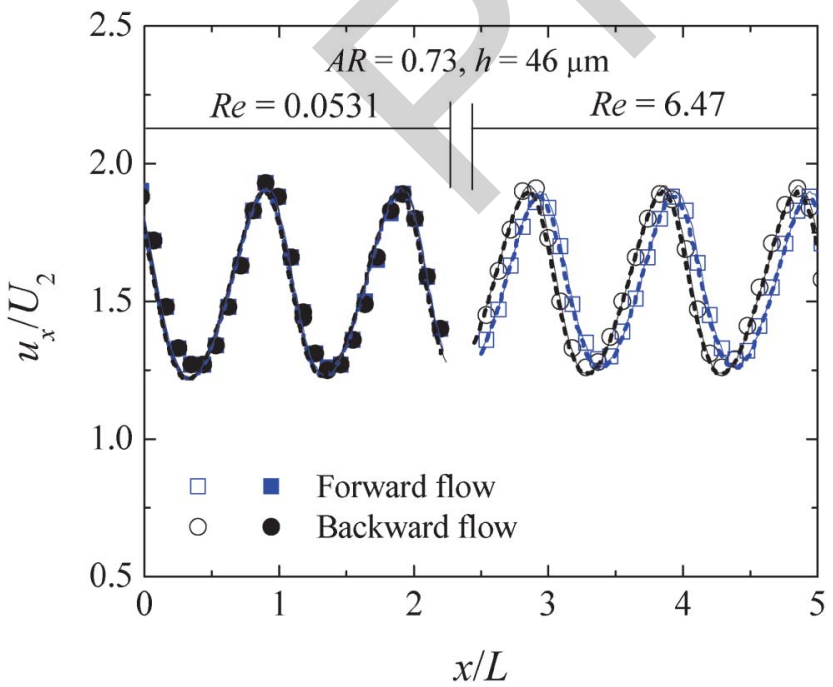

Fig. 4 Dimensionless axial velocity along the centreline for Newtonian fluid flow in the hyperbolic rectifier A $(A R=0.73, h=46 \mu \mathrm{m})$. Symbols: experimental data; dashed lines: numerical predictions using mesh M1; solid lines: numerical predictions using mesh M2. (left part of Fig. 4). When the flow rate is further increased, differences between the velocity profiles measured for the forward and backward flow directions become noticeable due to inertial effects (right part of Fig. 4) and, it is possible to identify a lag between both profiles. For the forward flow direction inertia pushes the fluid against the hyperbolic (gradual) contraction and the value of the velocity is maximum at the channel throat. On the other hand, for the backward flow direction, the fluid experiences a sudden acceleration when flowing through the abrupt contraction and the maximum value of the axial velocity occurs immediately downstream of the throat. Moreover, it is possible to observe that the amplitude of the velocity oscillation (and consequently the maximum strain rate) in the backward direction is slightly higher than in the forward flow direction. The numerical results presented in Fig. 4 were determined at the centreplane of the computational domain. However, since the $\mu$ PIV measurements take into account not only the focused particles at the centreplane but also unfocused particles at adjacent planes over the whole measurement depth, ${ }^{30}$ as described in Section 2.1, a numerical weightedaverage velocity in the measurement depth, $\delta z_{m}$, was calculated and a deviation from the centreline velocity field of less than $3 \%$ was found for rectifier A. For rectifier C, due to the larger depth, this deviation is significantly lower. Since the imparted difference in velocity is small and considering that the focused particles are responsible for the major contribution to the $\mu \mathrm{PIV}$ correlation, the presented numerical axial velocity is taken only at the centreline to compare with the experimental results. As can be seen in Fig. 4, the experimental and numerical results are in good agreement with each other. Moreover, the predictions obtained using meshes M1 and M2 are very similar and deviations between them are smaller than $1 \%$. For this reason, only mesh 

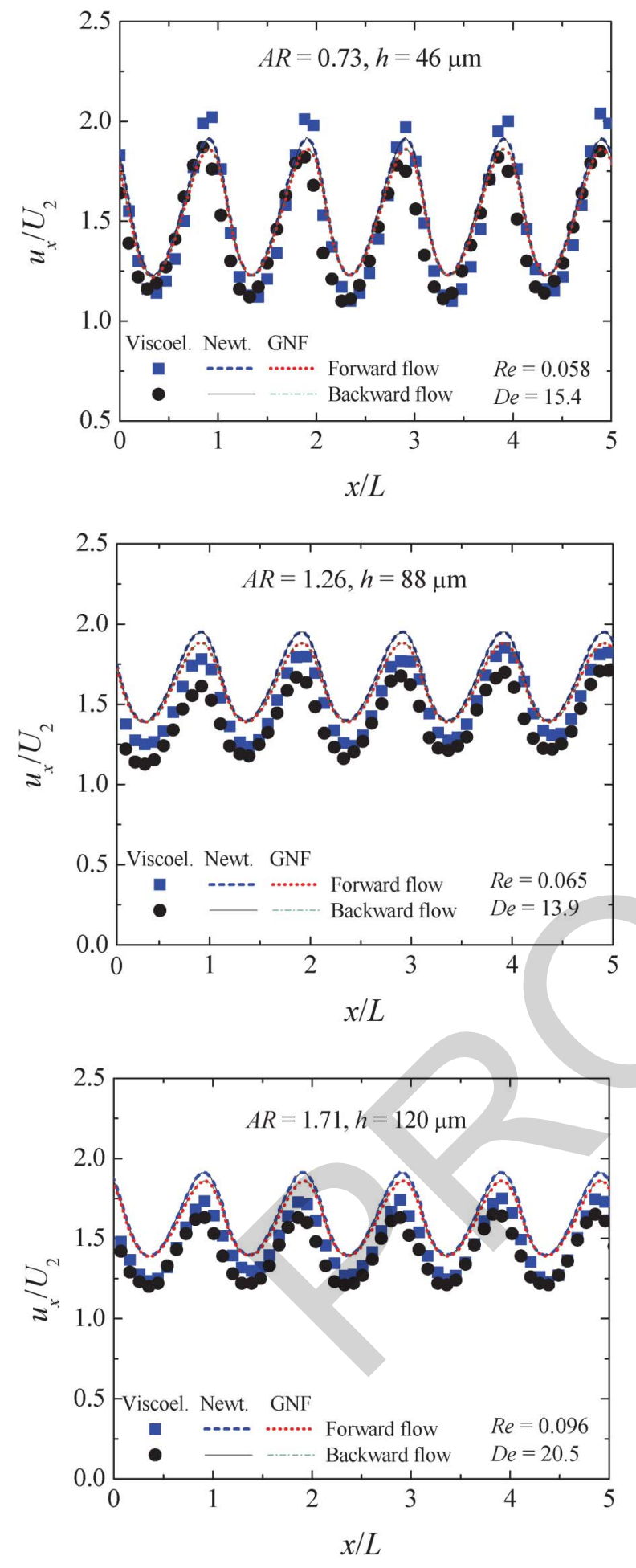

Fig. 5 Dimensionless axial velocity profiles along the centreline of the hyperbolic rectifiers with different aspect ratios under steady flow conditions. Symbols: experimental data for the viscoelastic fluid; lines: numerical predictions for a Newtonian and a generalized Newtonian fluid, described by the Carreau-Yasuda model, flowing at the same flow rate as the viscoelastic fluid.

M1 was used in the remaining simulations to allow for smaller CPU computational time required to converge the threedimensional simulations.

\subsection{Viscoelastic fluid flow}

As shown in Section 4.1, at low flow rates the flow is steady, but above a critical flow rate, elastic instabilities arise and the flow becomes unsteady, initially for the forward direction and then, at significantly higher flow rates, also in the backward flow direction.

a) Steady flow. Fig. 5 shows the axial velocity profiles measured with the viscoelastic fluid in all microgeometries investigated. The flow rates presented refer to steady flow conditions corresponding to similar flow resistance for both flow directions. Additionally, in Fig. 5 we also show the numerical predictions for a Newtonian fluid and a Carreau-Yasuda model, which exhibits a rheological shear behaviour similar to the viscoelastic fluid used in the experiments ( $c f$. model parameters in Section 2.2), flowing at the same flow rate as the viscoelastic fluid.

The velocity profiles exhibit the expected sinusoidal-like shape, with the experimental profile for the forward direction showing slightly higher amplitude, which is more evident for the channels with lower depths in contrast to what was observed for Newtonian fluids. Moreover, the forward and backward experimental velocity profiles also show a small lag in the $x$-direction, mostly due to elastic effects as is clear from Fig. 5(a) where $R e$ is too low for inertial effects to be relevant. Furthermore, it is also possible to observe that, for the rectifiers with a higher depth, the experimental velocity profiles are shifted below those predicted numerically, highlighting the presence of elastic effects on the experimental results, which are not captured in the numerical simulations using inelastic models.

b) Unsteady flow. The velocity field was also measured using $\mu \mathrm{PIV}$ at flow conditions for which the behaviour in the two flow directions is markedly different, i.e. when rectification effects are significant. Two flow rates were investigated for each microchannel, which correspond to the following scenarios: (i) unsteady flow in the forward direction and steady flow in the backward direction; (ii) unsteady flow in both forward and backward directions, corresponding to a higher flow rate.

When the flow becomes unsteady, due to the onset of an elastic instability, the vortices appear and disappear along time in some elements of the rectifier and in this case instantaneous velocity measurements are more suitable to analyse the flow behaviour. However, time-averaged measurements over a time scale significantly higher than the fluid relaxation time are also useful to establish the overall time-averaged flow field. In $\mu$ PIV measurements, the particle image density is generally low and high background noise is commonly observed, which means that using a single pair of images is generally not sufficient, even under steady conditions, to obtain accurate velocity measurements. ${ }^{36}$ Therefore, a correlation function based on averaging a high number of image pairs is usually used in order to improve the accuracy of the $\mu$ PIV measurements. ${ }^{30}$

In Fig. 6, we compare one instantaneous velocity profile (determined from two image pairs) with the average velocity described above (i.e. steady backward flow and unsteady forward flow). The unsteady nature of the forward flow is visible in the instantaneous velocity profile in Fig. 6, obtained at

10 
a given instant in time, since a clearly cyclic velocity profile is not observed. We note that the instantaneous velocity profile at different times is markedly different, due to the highly unsteady and irregular nature of the flow. However, the spatial periodic nature of the time averaged flow becomes clear with a large set of data collected.

On the other hand, the backward flow for the same flow rate remains steady as can be attested from the instantaneous velocity profile. Here, the axial velocity profile determined from two pairs of images acquired at a given instant in time is similar to the average velocity obtained by averaging 150 pairs of images, with deviations of about $10 \%$. In this same steady case, if the axial velocity profile is obtained from just 10 pairs of images, the deviation decreases to less than $5 \%$ when compared to that obtained from 150 pairs of images, whereas in the forward flow direction, the results obtained with 10 image pairs still present a disorderly pattern and we have used 1000 images pairs when calculating the time-averaged measurements for establishing the time-averaged flow field along the centreline. It is also interesting to note that the dimensionless velocity profile shown in Fig. 6 for the backward flow direction shows a significant velocity overshoot in the throat region $\left(u_{x} / U_{2} \approx 2.3\right.$ as compared with $u_{x} / U_{2}$ $\approx 1.9$ for lower $D e$ ) and a moderate velocity undershoot. This behaviour is typical of highly elastic contraction and expansion flows, as discussed by Alves and Poole. ${ }^{37}$

For higher flow rates, the flow becomes unsteady in both directions (scenario ii). In order to establish the overall evolution of the flow field and compare low and high De data, in Fig. 7 we show the average axial velocity profiles obtained for the two scenarios and for $h=120 \mu \mathrm{m}$. For unsteady flow conditions, the profiles were obtained using an average of 1000 image pairs. For the lower De cases (scenario i), the difference in the amplitude of oscillation between the velocity profiles for both flow directions is notorious, in accordance with the results shown in Fig. 6.

For the backward direction, in which the flow remains steady, the velocity gradient at the centreline is higher than for the forward direction. Since, in the latter, we are averaging pairs of images acquired over a long period of time, in which the flow

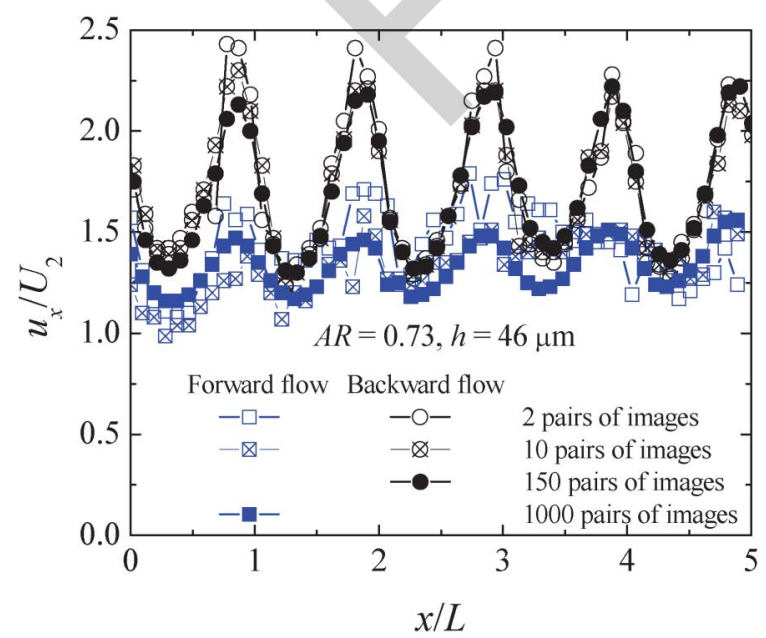

Fig. 6 Time-average and instantaneous dimensionless axial velocity profiles along the centreline of the hyperbolic rectifier A $(h=46 \mu \mathrm{m})$ for $R e=0.13$ and $D e=32.9$ (scenario i).

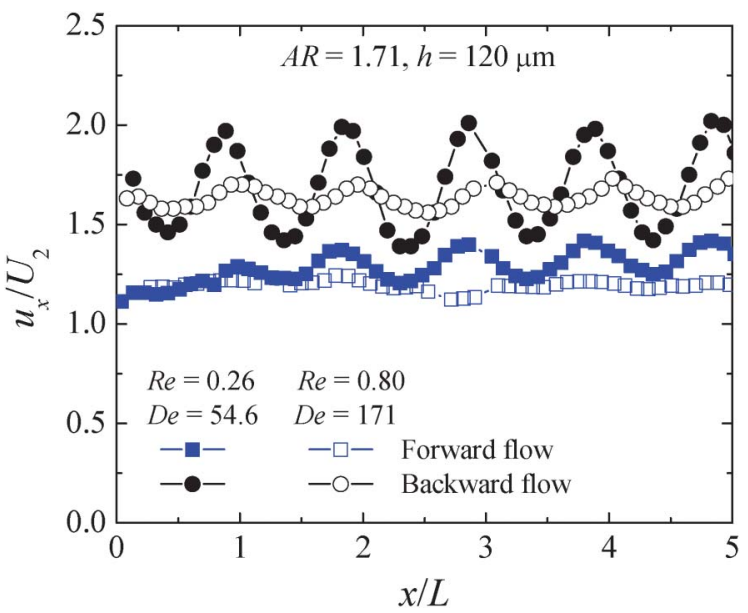

1

Fig. 7 Dimensionless axial velocity profiles along the centreline of the hyperbolic rectifiers with $h=120 \mu \mathrm{m}$ for the viscoelastic fluid flow. At the lower $D e$, the flow is unsteady in the forward flow direction and steady in the backward flow direction. At the higher $D e$ cases, the flow is unsteady in both flow directions.

behaviour was varying due to an elastic instability, the velocity fluctuations are smoothed out. At the higher De (cf. Fig. 7), when the flow is unsteady in both forward and backward directions, the amplitude of the average dimensionless velocity profile is lower than for lower elasticity flows especially for the backward flow direction. The differences between oscillating time-average forward and backward profiles are still noticeable, but smaller than at lower $D e$. For the forward flow direction, a more plug-like velocity profile is observed for both $D e$.

\subsection{Wall effect on the diodicity}

Fig. 8(a) shows the pressure drop per element of the rectifier measured experimentally as well as the corresponding numerical predictions for the Newtonian fluid flow through the hyperbolic rectifiers with different depths. Fig. 8(b) shows the pressure drop measured experimentally for the viscoelastic fluid. For both cases, the results obtained for the two flow directions are presented and are used to determine the variation of the diodicity of each microfluidic rectifier with $D e$.

As expected, for the same flow rate, the pressure drop observed in the microchannel with a smaller depth is significantly higher than that found for the deeper microchannel. For the Newtonian fluid, the pressure drop increases linearly with the flow rate, a consequence of the negligible inertial effects (in all cases $r^{2} \geqslant 0.991$, where $r$ is the sample correlation coefficient) .

Furthermore, in agreement with the findings of Sousa et al. ${ }^{27}$ negligible rectification effects are observed experimentally, i.e. for the same value of the flow rate, the corresponding forward and backward flow pressure drops are very similar, within experimental uncertainty. A good agreement between experimental and numerical results is observed. At the highest flow rates for the rectifier with $h=46 \mu \mathrm{m}$, small deviations are observed between the experimental data and the numerical predictions, but this is primarily due to the progressive deformation of the cross section area of the microchannels due to the high pressure differences required to obtain the higher flow rates (above 1.5 bar) and the corresponding deformation of the PDMS 

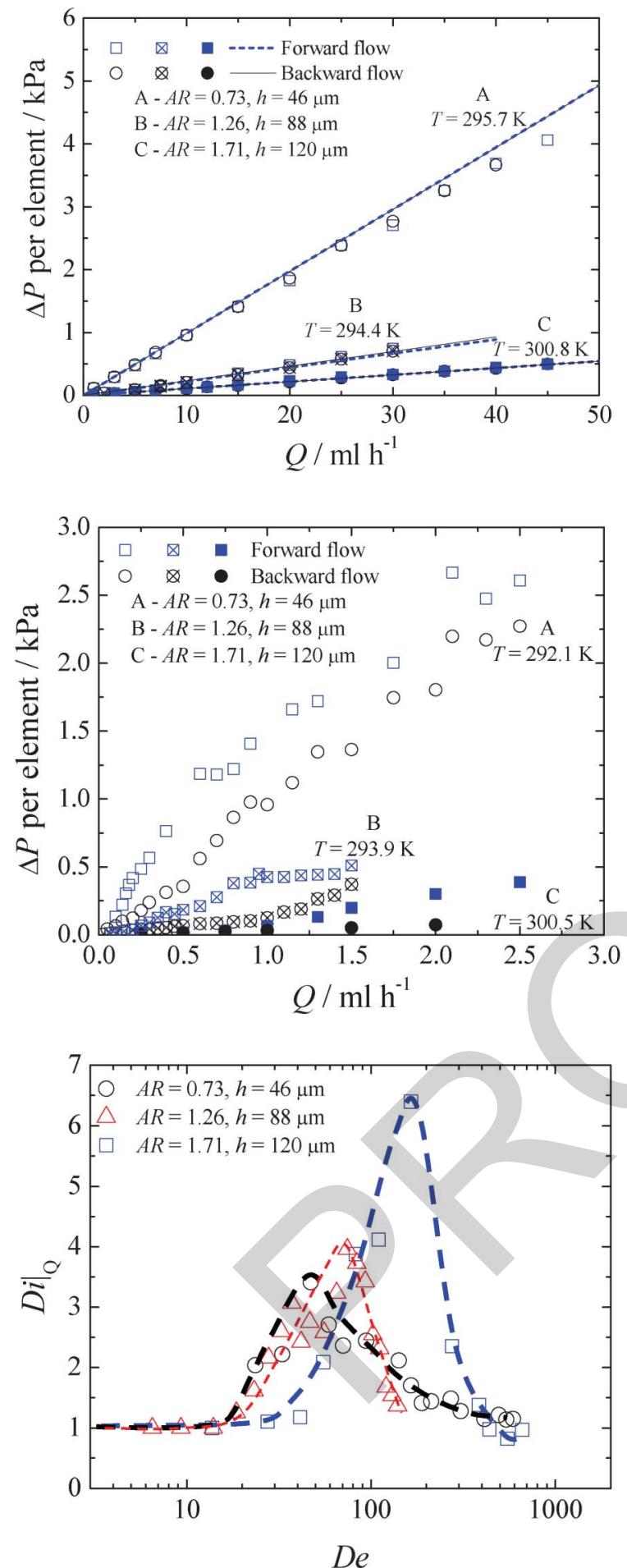

50 Fig. 8 Variation of the pressure drop per element of the rectifier, as a function of the flow rate and channel depth: (a) Newtonian fluid (symbols: experimental data; lines: numerical predictions), (b) viscoelastic fluid. (c) Diodicity as a function of Deborah number for the hyperbolic shaped rectifier with different depths. The dashed lines shown are a guide to the eye.

microchannels, ${ }^{38}$ leading to a progressively smaller experimental flow resistance as the flow rate increases.

For the non-Newtonian fluid flow, despite the considerably lower flow resistance measured in the deeper channel, in qualitative terms the overall behaviour is independent of the channel depth. At low flow rates, the pressure drop increases linearly with the flow rate, indicating a quasi-Newtonian flow behaviour. Increasing the flow rate (or $D e$ ) beyond a critical value, the flow resistance becomes considerably different for the two flow directions, which is directly related with the onset of an elastic instability in the forward flow direction. ${ }^{24,27}$ Above the critical flow rate, the flow resistance in the forward direction increases initially abruptly and then moderately as the flow rate increases. On the other hand, in the backward flow direction, the more pronounced increase of the flow resistance with the flow rate occurs only at significantly higher flow rates, since elastic instabilities in the backward flow direction also appear at higher flow rates. Hence, it is possible to infer, once again, that the changes in flow dynamics triggered by the onset of elastic instabilities lead to a markedly different flow resistance behaviour for both flow directions.

To better analyse the effect of the channel depth on the diodicity of the rectifier, in Fig. 8(c) we plot the diodicity, defined in terms of the pressure drop ratio for a constant flow rate, as a function of $D e$ for the three channel depths analysed.

The flow conditions corresponding to the maximum diodicity depend significantly on the aspect ratio of the channel as shown in Table 2.

As $A R$ increases the Deborah number at which the peak diodicity was obtained also increases from about $D e=45$ for geometry A to $D e=75$ for geometry $\mathrm{B}$ and $D e=165$ for microchannel $\mathrm{C}$. For microchannel $\mathrm{A}$, having the lowest aspect ratio $(A R=0.73 ; h=46 \mu \mathrm{m})$, the maximum diodicity is about 3.5. For the intermediate microchannel B $(A R=1.26 ; h=88 \mu \mathrm{m})$ the maximum diodicity is about 4 , while for the channel with the largest aspect ratio $(A R=1.71 ; h=120 \mu \mathrm{m})$ the maximum diodicity is about 6.4 . These values of diodicity are well above those previously achieved by other researchers. ${ }^{24,26}$

Thus, higher aspect ratios lead to higher rectification effects, suggesting that deeper geometries, where wall effects (or shear effects) are less pronounced and extensional flow is enhanced, may be preferred in order to devise efficient microfluidic diodes. It would be desirable to further increase the depth of the microfluidic diode, to investigate if higher diodicity would be achieved. However, using PDMS only moderate aspect ratio geometries can be replicated and other fabrication techniques need to be used to allow for precise fabrication of deeper microchannels. In any case, the map of diodicity displayed in Fig. 8(c), is valuable to determine the best rectifier for a wide range of flow rates, or at least of the best compromise between rectification effect and range of operation.

\section{Conclusions}

The flow of a Newtonian fluid (de-ionized water) and a nonNewtonian fluid with viscoelastic behaviour was investigated in

Table 2 Values of the Deborah number corresponding to the maximum diodicity for the three microchannels with different aspect ratios

\begin{tabular}{lrl}
\hline$A R$ & $D e$ & Maximum $\left.D i\right|_{\mathrm{Q}}$ \\
\hline 0.73 & 45 & 3.4 \\
1.26 & 75 & 4.0 \\
1.71 & 165 & 6.4 \\
\hline
\end{tabular}


1 microfluidic rectifiers with hyperbolic shape to assess the effect of channel depth (aspect ratio) upon their diodicity. The Newtonian fluid flow was also simulated numerically and a good agreement with the experimental results was found. The fluid flow behaviour is qualitatively similar in the microgeometries with different depths. For the Newtonian fluid, the axial velocity at the centreline shows a periodic pattern due to geometric effects. At low inertial flow conditions, the velocity profile in the forward flow direction resembles that in the 0 backward flow direction, while increasing the flow inertia leads to the appearance of a spatial offset between both velocity profiles. For the viscoelastic fluid, different flow characteristics were found. At low $D e$, the velocity profiles are Newtonian-like, but upon increasing the flow rate, the flow becomes unsteady and the axial velocity along the centreline of the microchannel varies in time, due to the onset of an elastic instability, which occurs at a lower $D e$ in the forward flow direction than in the backward flow direction.

The measured pressure drop through the hyperbolic rectifiers 20 shows a similar pattern for all $A R$. For the Newtonian fluid, the experimental results do not reveal noticeable rectification effects. In contrast, for the viscoelastic fluid, an anisotropic flow resistance is observed independently on the aspect ratio of the microchannel, but at different flow rate ranges. In terms of diodicity, the microfluidic rectifier with the highest aspect ratio presented a maximum diodicity of about 6.4 , which is clearly in excess any other work reported in the literature The results suggest that, to some extent, reduction of shear and enhancement of the extensional component of the flow field (achieved by decreasing the effect of bounding walls) leads to higher diodicity.

\section{Acknowledgements}

The authors acknowledge the financial support provided by Fundação para a Ciência e a Tecnologia (FCT) and FEDER through projects PTDC/EQU-FTT/71800/2006, REEQ/262/ EME/2005 and REEQ/928/EME/2005. P. C. Sousa would also like to thank FCT for financial support through scholarships SFRH/BD/28846/2006 and SFRH/BPD/75258/2010.

\section{References}

1 G. M. Whitesides, Nature, 2006, 442, 368-373.

2 R. Gómez-Sjöberg, A. A. Leyrat, D. M. Pirone, C. S. Chen and S. R. Quake, Anal. Chem., 2007, 79, 8557-8563.

3 J. O. Tegenfeldt, C. Prinz, H. Cao, R. L. Huang, R. H. Austin, S. Y. Chou, E. C. Cox and J. C. Sturm, Anal. Bioanal. Chem., 2004, 378, 1678-1692.

4 E. Verpoorte, Electrophoresis, 2002, 23, 677-712.
5 J. deMello, Nature, 2006, 442, 394-402.

6 H. A. Stone and S. Kim, AIChE J., 2001, 47, 1250-1254.

7 K. L. Helton and P. Yager, Lab Chip, 2007, 7, 1581-1588.

8 F. J. Tovar-Lopez, G. Rosengarten, E. Westein, K. Khoshmanesh, S. P. Jackson, A. Mitchell and W. S. Nesbitt, Lab Chip, 2010, 10, 291-302.

9 S. Mall-Gleissle, W. Gleissle, G. H. McKinley and H. Buggisch, Rheol. Acta, 2002, 41, 61-76.

10 M. S. N. Oliveira and G. H. McKinley, Phys Fluids (Letters), 2005, 17, 071704.

11 S. J. Muller, R. G. Larson and E. S. G. Shaqfeh, Rheol. Acta, 1989, 28, 499-503.

12 R. G. Larson, E. S. G. Shaqfeh and S. J. Muller, J. Fluid Mech., 1990, 218, 573-600.

13 P. Pakdel and G. H. McKinley, Phys. Rev. Lett., 1996, 77, 2459.

14 P. E. Arratia, C. C. Thomas, J. Diorio and J. P. Gollub, Phys. Rev. Lett., 2006, 96, 144502.

15 R. J. Poole, M. A. Alves and P. J. Oliveira, Phys. Rev. Lett., 2007, 99, 164503.

16 J. A. Pathak and S. D. Hudson, Macromolecules, 2006, 39, 8782-8792.

17 J. Soulages, M. S. N. Oliveira, P. C. Sousa, M. A. Alves and G. H. McKinley, J. Non-Newtonian Fluid Mech., 2009, 163, 9-24.

18 M. S. N. Oliveira, F. T. Pinho, R. J. Poole, P. J. Oliveira and M. A. Alves, J. Non-Newtonian Fluid Mech., 2009, 160, 31-39.

19 H. Y. Gan, Y. C. Lam and N.-T. Nguyen, Appl. Phys. Lett., 2006, 88, 224103.

20 A. Groisman and V. Steinberg, Nature, 2000, 405, 53-55.

21 A. Groisman and V. Steinberg, Nature, 2001, 410, 905-908.

22 A. Groisman and V. Steinberg, New J. Phys., 2004, 6, 29-48.

23 A. Groisman, M. Enzelberger and S. R. Quake, Science, 2003, 300, 955-958.

24 A. Groisman and S. R. Quake, Phys. Rev. Lett., 2004, 92, 095401.

25 M. Navabi, Microfluid. Nanofluid., 2009, 7, 599-619.

26 N.-T. Nguyen, Y.-C. Lam, S. S. Ho and C. L.-N. Low, Biomicrofluidics, 2008, 2, 034101.

27 P. C. Sousa, F. T. Pinho, M. S. N. Oliveira and M. A. Alves, J. NonNewtonian Fluid Mech., 2010, 165, 652-671.

28 M. S. N. Oliveira, M. A. Alves, F. T. Pinho and G. H. McKinley, Exp. Fluids, 2007, 43, 437-451.

29 Y. Xia and G. M. Whitesides, Angew. Chem., Int. Ed., 1998, 37, $550-575$.

30 D. Meinhart, S. T. Wereley and M. H. B. Gray, Meas. Sci. Technol., 2000, 11, 809-814.

31 J. Dealy and D. Plazek, Rheology Bulletin, 2009, 78, 16-31.

32 N. Phan-Thien and R. I. Tanner, J. Non-Newtonian Fluid Mech., 1977, 2, 353-365.

33 P. J. Oliveira, F. T. Pinho and G. A. Pinto, J. Non-Newtonian Fluid Mech., 1998, 79, 1-43.

34 M. A. Alves, P. J. Oliveira and F. T. Pinho, Int. J. Numer. Methods Fluids, 2003, 41, 47-75.

35 R. B. Bird, R. C. Armstrong, O. Hassager, Dynamics of polymeric liquids. Volume 1: Fluid Dynamics, John Wiley \& Sons, 1987.

36 S. T. Wereley and C. D. Meinhart, Micron-resolution particle image velocimetry in micro- and nano-scale diagnostic techniques, SpringerVerlag, 2005.

37 M. A. Alves and R. J. Poole, J. Non-Newtonian Fluid Mech., 2007, 144, 140-148.

38 S. Hardy, K. Uechi, J. Zhen and H. P. Kavehpour, Lab Chip, 2009, 9 , 935-938. 